\title{
Growing up: the role of the Royal College of Physicians
}

\author{
Janet E McDonagh and Angela J Robinson
}

\section{Introduction}

The health of young people as distinct from that of children and adults has recently received increasing recognition in national policies and guidance. However, the engagement of healthcare professionals to lead the necessary changes remains a challenge. This editorial presents the evidence to advocate a crucial role for the Royal College of Physicians in the healthcare of adolescents and young adults in the UK today.

\section{Background}

Adolescence is a time of adoption of health-promoting and health-risking behaviours, both of which have the potential to influence future adult health and ill-health. Adolescents have specific health needs related to their developmental status, which in turn need to be acknowledged and appropriately addressed by healthcare providers. While the developmental stage of adolescence commences within the paediatric arena, neuroscientific advances reveal that adolescent brain development continues into the mid twenties, ${ }^{1}$ which places adult healthcare in a pivotal role. Psychosocial adolescent development likewise continues into the twenties. In the UK, $80 \%$ of young men aged 18-24 years are still living in the parental home. ${ }^{2}$ The evidence suggests that $41.6 \%$ of young adults (18-30-year-olds) do not self-classify themselves as adults. ${ }^{3}$ Although 'young adults' may have similar logical competencies as older adults, their different psychosocial status influences their decision-making and risk-taking. There is therefore a need to adopt a developmentally appropriate approach in adult care whenever adolescents and young adults are encountered.

\section{Epidemiology}

The number of adolescents aged 10-19 living in the UK has increased by more than $11 \%$ since 1995 to around 7.6 million today; ${ }^{2} 16-24$-year-olds account for $12 \%$ of the resident UK population. $^{2}$ The perception may be that members of these groups are young, fit and healthy, but their use of health services is significant. Between 1996 and 2010, emergency admissions among the 16-19-year-old age group increased by a dramatic $43 \% .{ }^{2}$ Hospital admissions among 10-19-year-olds for

Janet McDonagh, clinical senior lecturer in paediatric and adolescent rheumatology and clinical lead for adolescent health, University of Birmingham \& Birmingham Children's Hospital NHS Foundation Trust; Angela J Robinson, consultant in genitourinary medicine, Mortimer Market Centre, London. diabetes, asthma and epilepsy, the three most common longterm conditions affecting young people, have increased by over $26 \%$ over the past eight years. ${ }^{2}$ In adolescent-onset cancer, mortality rates have failed to see the improvements observed in childhood and adult cancer. ${ }^{4}$ Although new diagnoses of chlamydia have shown a marginal decrease since 2008, this, along with other sexually transmitted infections, remains a significant public health issue for 16-24-year-old men and women in the UK. ${ }^{2}$

For young people with childhood-onset long-term conditions, the event of transfer to adult care can be associated with significant morbidity. Graft loss and/or rejection-related mortality for adolescent and young adult transplant recipients is reported to be worse than for any other age-group. ${ }^{5}$ Similarly, there is an increased rate of hospitalisation post-transfer reported in diabetes. ${ }^{6}$ Loss to and/or lack of follow-up following transfer to adult services have been reported in several conditions, ${ }^{7,8}$ which leads to serious health consequences at great personal and societal cost. Reasons for lapses of care post transfer to adult services include lack of availability of adult specialist care, young people presuming cure, lack of health and disease literacy - an integral component of effective transition - and psychological reasons, all of which could be addressed with effective multidisciplinary transitional care to young adult healthcare.

With medical advances, more young people with childhoodonset conditions are surviving into adulthood. These include those with inherited metabolic disease, muscular dystrophy and certain types of congenital heart disease. Specific adult services for such conditions (including palliative care) are at varying stages of development in the UK. With continuing medical and technological advances, effective planning involving both paediatric and adult care providers, including physicians, from a range of disciplines will be vital to ensure that developmentally appropriate service provision keeps pace with survival rates. For many conditions, there will also be young people diagnosed in late adolescence and young adulthood who have never experienced paediatric care, further highlighting the importance of ensuring the provision of care that is appropriate to age and developmental stage, beyond the event of transfer to adult services.

\section{Current adolescent and young adult healthcare}

Paediatric and adult healthcare are two very dissimilar systems with different cultures of care, and therein lies the challenge of transition irrespective of the specific disease. Acknowledging these differences as professionals ${ }^{9}$ and preparing young people and their families for them is vital. This entails collaboration 
across the paediatric and adult interface so that both services are aware of each other's provision. Despite high levels of overall satisfaction, young adults report a poorer experience of care than older adults according to UK survey data. ${ }^{10}$ In a recent editorial entitled Young adults are worse off than adolescents, the changes in healthcare utilisation and their negative impact on the health of young adults were highlighted. ${ }^{11}$ Although US-based data were presented, the increased emergency admission rate observed in the UK for the 16-19 age group in the UK may reflect similar factors at play. ${ }^{2}$ The 'You're welcome' quality criteria for young-person-friendly services, ${ }^{12}$ endorsed by the World Health Organisation and now revised for use in secondary and tertiary hospital-based services, is a much-needed set of quality indicators. These will prove useful in objective assessment of health service provision for this age group.

There is a growing body of evidence to support the development of young adult healthcare services for the 16-25-year-old age group. Young adult clinics were identified as a key strategy of successful transition programmes in a recent systematic review. ${ }^{13}$ Bent et al reported that a young adult multidisciplinary team approach for young people with disabilities cost no more to implement than an ad hoc approach and was actually more likely to enhance participation of involved young people in society. ${ }^{14}$ Several specialties are already recognising the young adult age group as needing more innovative ways to access services, reflected by the current NHS Kidney Care Supporting young adults with renal disease project. ${ }^{15}$ The young adult clinic concept is particularly appropriate to address the discrepancy between average age and/or case mix of patients between paediatric and adult clinics, and potentially facilitates uninterrupted service provision of age and developmentally appropriate care alongside the other key adolescent transitions, such as vocation, independent living, etc. Finally, young adult clinics can facilitate adolescent healthcare provision within adult services and potential paediatric-adult collaboration in terms of clinical service, education and training, research and the process of transition.

\section{Training issues}

Until the launch of the Department of Health-funded e-learning Adolescent Health Project, ${ }^{16}$ there was no formal adolescent health training outside mental health in the UK. Acknowledging the reported unmet adolescent health training needs of paediatric professionals, ${ }^{17}$ Okumura et al reported that adult physicians were more likely than paediatricians to report that insufficient training limited their ability to provide care for young people with chronic conditions. ${ }^{18}$ The time is therefore ripe for joint Royal College training initiatives, particularly with respect to the core topics of relevance to any specialist encountering young people in adult services (see Box 1).

\section{Conclusions}

With development extending beyond the normal age criteria for paediatrics, the delivery of developmentally appropriate services
Box 1. Core modules in adolescent health for adult specialty training programmes.

Healthy development

Legal framework

Communication and consultation with young people

Chronic conditions and transition

Concordance and adherence

Common medical conditions

Implications of health-risk behaviours

Youth-friendly health services

for adolescents and young adults will require collaboration between paediatric and adult healthcare professionals. A change in mindset from the dichotomous model of paediatrics and adult medicine towards the integration of adolescent health into both is urgently needed in the NHS and clinical research programmes. The argument is not for the formation of yet another specialty, but rather for health professionals with adolescent expertise to exist wherever young people are seen in the NHS. Young adults and adolescents should expect high quality healthcare, but evidence suggests that their needs are not met by current provision. Physicians must acknowledge the shortfall and the Royal College of Physicians can provide vital leadership in identifying the issues, developing standards in collaboration with other Royal Colleges and implementing the necessary changes.

\section{References}

1 Steinberg L. A behavioral scientist looks at the science of adolescent brain development. Brain Cogn 2010;72:160-4.

2 Coleman J, Brooks F, Treadgold P. Key data on Adolescence 2011 ( $8^{\text {th }}$ edition). London: AYPH, 2011.

3 Luyckx K, Moons P, Weets I. Self-classification as an adult in patients with type 1 diabetes: relationships with glycemic control and illness coping. Patient Educ Couns 2011:85,245-50.

4 Albritton K, Bleyer WA. Young adult oncology: the patients and their survival challenges. Eur J Cancer 2003;39:2584-99.

5 George JF, Taylor DO, Blume ED et al. Minimising infection and rejection death: clues acquired from 19 years of multi-institutional cardiac transplantation data. J Heart Lung Transplant 2011;30:151-7.

6 Nakhla M, Daneman D, To T et al. Transition to adult care for youths with diabetes mellitus: findings from a Universal Health Care System. Pediatrics 2009;124:e1134-41.

7 Klosky JL, Cash DK, Buscemi J et al. Factors influencing long-term follow-up clinic attendance among survivors of childhood cancer. Cancer Surviv 2008;2:225-32.

8 Yeung E, Kay J, Roosevelt GE et al. Lapse of care as a predictor for morbidity in adults with congenital heart disease. Int J CArdiol 2008;125:62-5.

9 Tattersall R, McDonagh JE. Transition: a rheumatology perspective. $\mathrm{Br}$ J Hospital Medicine 2010;71:315-319.

10 Hargreaves DS, Viner RM. Children's and young people's experience of the National Health Service in England: a review of national surveys 2001-2011. Arch Dis Child 2011. [Epub ahead of print] 
11 Callahan ST, Cooper WO. Changes in ambulatory health care use during the transition to young adulthood. J Adolesc Health. 2010;46:407-13.

12 Department of Health. You're welcome quality criteria. Making health services young people friendly. London: DH, 2011.

13 Crowley R, Wolfe I, Lock K, McKee M. Improving the transition between paediatric and adult healthcare: a systematic review. Arch Dis Child 2011;96:548-53.

14 Bent N, Tennant A, Swift T et al. Team approach versus ad hoc health services for young people with physical disabilities: a retrospective cohort study. Lancet 2002;360:1280-86.

15 NHS Kidney Care. Supporting Young Adults with Renal Disease. www.kidneycare.nhs.uk/_ourworkprogrammes-preparationsupportingyoungadultswithkidneydisease.aspx. [Accessed 26 January 2012].

16 Royal College of Pediatrics and Child Health. Adolescent Health Programme. www.rcpch.ac.uk/ahp. [Accessed 25 January 2012].
17 McDonagh JE, Minnaar G, Kelly KM et al. Unmet education and training needs in adolescent health of health professionals in a UK Children's Hospital. Acta Paediatr 2006;95:715-9.

18 Okumura MJ, Heislef M, Davis MM et al. Comfort of general internists and general pediatricians in providing care for young adults with chronic illnesses of childhood. J Gen Intern Med 2008;23:1621-7.

Address for correspondence: Dr J McDonagh,

University of Birmingham \& Birmingham Children's

Hospital NHS Foundation Trust,

Steelhouse Lane, Birmingham B4 6NH.

Email: j.e.mcdonagh@bham.ac.uk

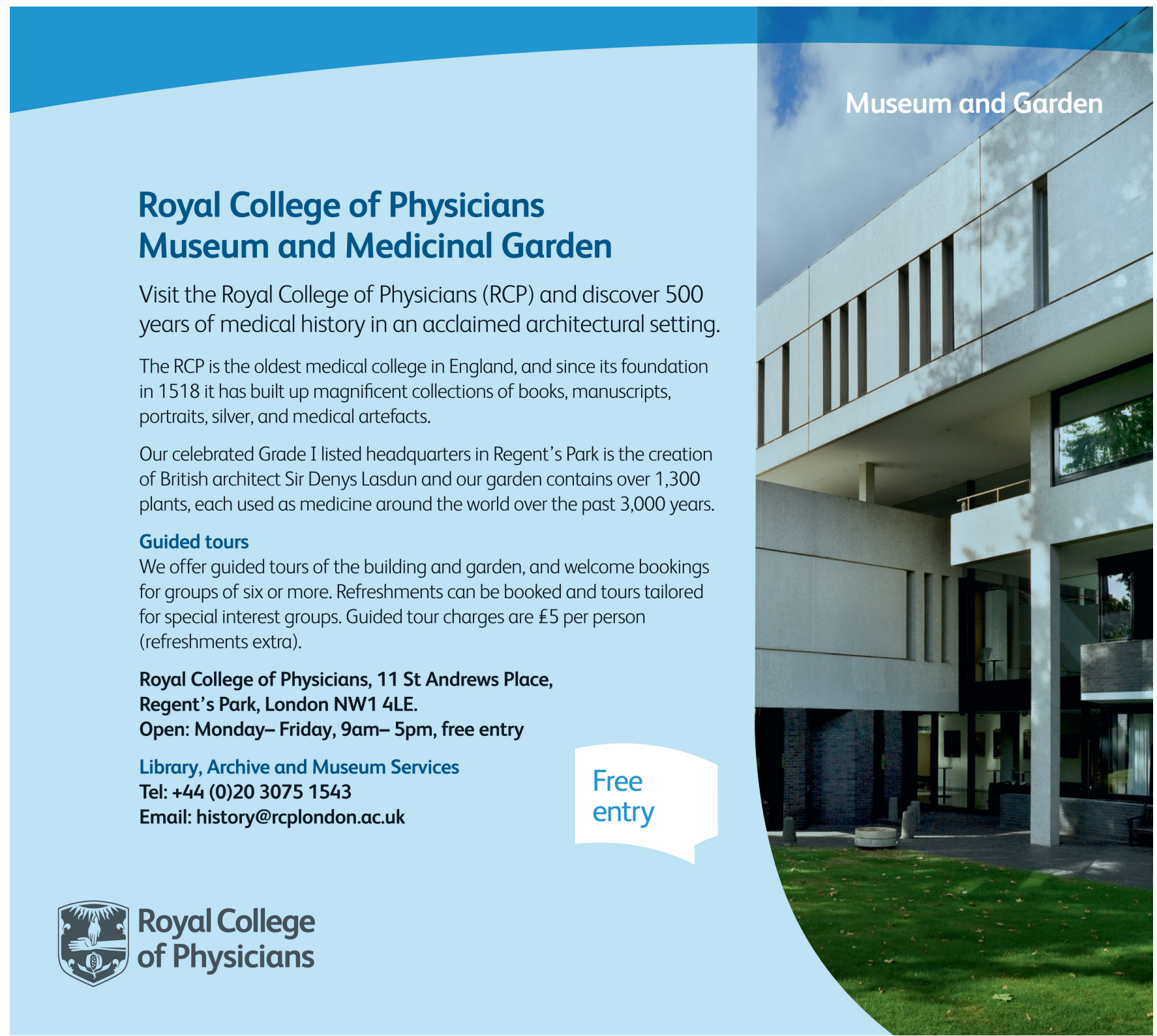

